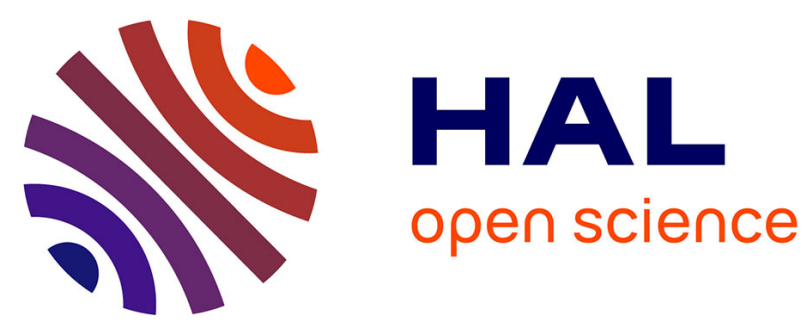

\title{
IDENTIFICATION DE LA NATURE DU FOND DE LA MER À L'AIDE DE SIGNAUX D'ÉCHO-SONDEURS
}

\author{
E. Pouliquen, X. Lurton
}

\section{- To cite this version:}

E. Pouliquen, X. Lurton. IDENTIFICATION DE LA NATURE DU FOND DE LA MER À L'AIDE DE SIGNAUX D'ÉCHO-SONDEURS. Journal de Physique IV Proceedings, 1992, 02 (C1), pp.C1941-C1-944. 10.1051/jp4:19921205 . jpa-00251171

HAL Id: jpa-00251171

https://hal.science/jpa-00251171

Submitted on 1 Jan 1992

HAL is a multi-disciplinary open access archive for the deposit and dissemination of scientific research documents, whether they are published or not. The documents may come from teaching and research institutions in France or abroad, or from public or private research centers.
L'archive ouverte pluridisciplinaire HAL, est destinée au dépôt et à la diffusion de documents scientifiques de niveau recherche, publiés ou non, émanant des établissements d'enseignement et de recherche français ou étrangers, des laboratoires publics ou privés. 


\title{
IDENTIFICATION DE LA NATURE DU FOND DE LA MER À L'AIDE DE SIGNAUX D’ÉCHO-SONDEURS
}

\author{
E. POULIQUEN et X. LURTON \\ IFREMER, Centre de Brest, DITI/SM/ASM, BP. 70, F-29280 Plouzane, France
}

\begin{abstract}
RESUME: On présente une méthode originale d'identification en temps réel de la nature des fonds marins superficiels du plateau continental à partir de l'analyse de l'enveloppe temporelle des signaux de d'échos-sondeurs du type de ceur utilisés en bathymétrie ou en péche. Le principe consiste d̀ comparer les caractéristiques temporelles du signal réverbéré avec des signaux de référence définis pour un sondeur donné et sept types de fonds différents. La méthode a été validée in situ sur un grand nombre de configurations donnant des résultats très concluants.
\end{abstract}

ABSTRACT: A new method is proposed for real-time identification of the continental shelf seabed, using signal time envelopes of echo-sounders for bathymetry and fisheries. The method consists in comparing the temporal reverberated signal with a set of reference signals computed for a given sounder and a variety of water-heights and sea-bed types. The theoretical model for time reverberation is at first presented, based on classical approaches (tangent plane and small perturbations methods), and is validated on experimental echoes. The classification system is then described, together with the results of the at-sed validation campaigns.

\section{MODELE TEMPOREL DE REVERBERATION DE FOND POUR UN SONDEUR}

Les sondeurs standard de peche ou de bathymêtrie utilisent des signaux très simples (impulsions brèves monofréquence) de fréquence élevée (f > 30kHz) pour lesquels I'écho renvoyé par le fond est principalement du au relief d'interface eau-sédiment. Cependant, pour les fonds à faible granulométrie, la rétro-diffusion par les inhomogénéités de la couche superficielle des sédiments est à prendre en compte dans la réverbération totale. Ces contributions respectives de l'interface et du volume dépendent des caractéristiques physiques des fond étudiées (micro-relief, contraste d'impédance eau-fond, degré d'inhomogénéité du fond, absorption, angle d'incidence de 1'onde émise). Comme la réponse angulaire du fond est un facteur nettement classifiant, on propose de l'utiliser dans I'exploitation des signaux d'échos-sondeurs, dont les impulsions très brèves (de 0.1 a 1 ms) effectuent pratiquement en fonction du temps un balayage angulaire fin du fond intercepté par le faisceau du sondeur (fig.1). On a donc modélisé, en fonction du signal émis, l'enveloppe temporelle reçue par le sondeur après réverbération par différents types de fond. Ce modéle utilise 1'approche classique de l'approximation du plan tangent pour décrire la diffusion des ondes à I'interface eau-fond; 1 'indice de réverbération d'interface utilisé $(m(\Phi))$ est celui de Brekhovskikh [1]. La méthode des petites perturbations est utilisee pour introduire l'effet de diffusion de 1 'onde dans les sédiments; on a pour cette application repris l'indice de réverbération volumique $\left(d m_{y}(\Phi, r)\right)$ d'Ivakin \& Lysanov [2] adapté au cas d'une utilisation en mode impưlsionnel.

$L^{\prime}$ 'enveloppe temporelle $I_{f}(t)$ de $l^{\prime}$ intensité totale reçue par le sondeur dans le cas d'une impulsion carrée peut être décomposée selon les contributions $I_{s}(t)$ et $I_{v}(t)$ de l'interface et du volume du fond:

$$
I_{t}(t)=I_{s}(t)+I_{v}(t)=\int E(t) \cdot d^{2} I_{s}+\int E(t) \cdot d^{3} I_{v}
$$


où $d^{2} I_{s}$ et $d^{3} I_{v}$ sont les intensités élémentaires reçues par le sondeur provenant respectivement ${ }^{v} d^{\prime}$ un élement $d^{\prime}$ interface dS et $d^{\prime} u n$ élément de volume dS.dr:

$d^{2} I_{s}=D(\Phi, \Psi, \theta) \cdot m_{s}(\Phi) \cdot\left(e^{-2 \alpha R} / R^{2}\right)^{2} \cdot d S$ et $d^{3} I_{v}=D(\Phi, \Psi, \theta) \cdot d m_{v}(\Phi, r) \cdot\left(e^{-2 \alpha R} / R^{2}\right)^{2} \cdot d S$

$D(\Phi,, \theta)$ est la fongtign de directivité du sondeur, $\theta$ est 1 'angle d'inclinaison par rapport à la verticale, $\left(e^{-2 \alpha} / R^{2}\right)^{2}$ est le terme de pertes géométriques et d'absorption dans l'eau, $E(t)$ est la fonction d'insonification : $E(t)=1$ si $\Phi \in[\Phi(t-T, r), \Phi(t, r)]$ $=0$ sinon.

$\Phi(t, r)$ est l'angle localisant les éléments de surface et de volume par rapport à la verticale au sondeur contribuant au temps $t$ au signal reçu (fig.1):

$$
\Phi(t, r)=\operatorname{arcos}\left[h /\left(h+c t / 2-r \cdot c / c_{1}\right)\right]
$$

Dans le cas d'une impulsion de forme $F(t), I_{t}(t)$ devient:

$$
I(t)=\int_{0}^{\min (t, T)} F(\tau) \cdot I_{t}(t-\tau) \cdot d \tau
$$

Cependant, dans $I^{\prime}$ expression $(3)$ de $I(t)$, il $n^{\prime}$ est pas tenu compte de $I^{\prime}$ effet de retard dû aux irrégularités de hauteur d'eau. Ces variations par rapport à la hauteur moyenne $h$ ne sont pas du tout négligeables et entrainent des modifications de l'enveloppe $I(t)$ calculée précédemment. Si $l^{\prime}$ on décrit le relief du fond par une distribution $R(h), I$ expression $S(t)$ de l'enveloppe temporelle de l'intensité réverbérée devient en tenant compte de cet effet:

$$
S(t)=\int_{0}^{z(t)} I(t-2 \cdot z /(c \cdot \cos \theta)) \cdot R(z) \cdot d z
$$

où $z(t)=\min \left(c . t / 2,2 . h_{0}\right), h_{0}$ étant la valeur extrême du relief.

Les enveloppes ainsi calculées en choisissant les paramètres physiques appropriés pour plusieurs types de fond reproduisent bien la réalité expérimentale (fig.2). Toutefois les enveloppes mesurées sont très fluctuantes et il est difficile de les comparer directement au modèle: on présente ici des exemples d'échos temporels théoriques (fig.2) et expérimentaux (fig.3) correspondant à plusieurs types de fonds. On notera par ailleurs que les signaux temporels obtenus dépendent de manière complexe de la hauteur d'eau et du temps d'impulsion: un accroissement de ces paramètres ne provoque pas une simple distorsion d'échelle temporelle.

\section{CLASSIFICATION A PARTIR DES ENVELOPPES TEMPORELIES}

Une approche purement énergétique des échos réverbérés ne nous semble pas permettre de résoudre le problème de l'identification du fond: en effet la dynamique des variations de I'écho (soit son energie totale soit son niveau maximal) en fonction du type de fond est peu significative, et en tous cas inférieure aux fluctuations individuelles des échos sur un fond donné. Ayant établi que la forme temporelle des signaux de sondeurs possède un caractère suffisamment caractéristique du type de fond provoquant l'écho réverbéré, on cherche à utiliser cette propriété pour effectuer une classification automatique des échos obtenus par un sondeur. Le problème de I'utilisation de tels échos en situation opérationnelle étant, comme on l'a dit, leur caractère très fluctuant, on effectue un double traitement préliminaire:

- on considère non plus 1 'enveloppe temporelle du signal brut, mais l'intégrale de l'énergie reçue en fonction du temps, et normalisée par sa valeur maximale (fig.4):

$$
E(t)=\int_{0}^{t} S(\tau) \cdot d \tau / \int_{0}^{T \max } S(\tau) \cdot d \tau \quad t \in[0, T \max ]
$$

- on effectue Ie traitement d'identification sur plusieurs signaux $E(t)$ successifs et moyennés. 
L'intérêt d'utiliser l'énergie intègrée et normalisêe plutôt que le signal temporel brut est de pouvoir s'affranchir des fluctuations importantes du niveau maximal, et de lisser le profil assez chaotique des échos individuels. Le moyennage supplémentaire effectué permet d'obtenir des signaux encore plus stables, et n'est pas pénalisant dans la mesure où il est de toute manière inutile d'effectuer une opération d'identification du fond à chaque coup de sonde (c'est à dire à une cadence de l'ordre de la seconde).

Les signaux ainsi transformés peuvent alors être comparés à un jeu de courbes moyennes caractéristiques des principaux types de fonds rencontrés en zone littorale. Le nombre de ces signaux de référence dépend de la finesse que l'on souhaite pour la classification, mais surtout des possibilités du modèle et du traitement numérique. Dans la pratique on a retenu une classification en sept catégories (roche, gravier, sable, sable fin, vase-sable, vase, vase molle); cette classification permet une identification suffisament précise du fond, tout en évitant suffisamment les confusions entre classes voisines.

\section{MISE EN OEUVRE ET RESULTATS EXPERIMENTAUX}

On a mis au point pour la validation expérimentale de la méthode un logiciel permettant de traiter en temps réel des signaux temporels issus d'un système de numérisation disposé à la sortie du sondeur. Le logiciel effectue en phase préliminaire le calcul des signaux de référence (qui serviront à l'identification), puis au fur et à mesure de 1'acquisition des signaux, assure les fonctions suivantes:

- sélection de la portion du signal temporel correspondant à l'écho de fond.

- détermination de la hauteur locale approchée.

- pré-sélection de I'écho, qui sera rejeté si la mesure de la hauteur d'eau (front de montée du signal) est visiblement aberrante, ou si l'énergie totale du signal est trop différente de $I$ 'énergie moyenne des autres échos de la séquence.

- conditionnement de I'écho: recalage à partir du front de montée, calcul de l'intégrale de 1 'énergie cumulée.

- moyennage d'un nombre (5 ou 10) d'échos successifs ainsi conditionnés.

- comparaison de $I^{\prime}$ écho moyen avec les échos thériques des sept types de fond correspondant à la hauteur d'eau, et sélection du résultat.

L'essentiel des validations expérimentales a été effectué au cours de la campagne ETAP91 menée à bord du N.0. THALASSA en juillet 1991. Le système d'identification de fond a été couplé à un sondeur numérique, émettant des impulsions de $0,2 \mathrm{~ms}$ à $1,4 \mathrm{~ms}$ à $38 \mathrm{kHz}$. Des dizaines d'heures d'enregistrements systematiques ont été effectués, sur de nombreux fonds du plateau continental français; une trentaine de stations de prélèvement de sédiments superficiels, destiné à valider l'identification acoustique, ont été effectuées. Environ 200000 échos ont été acquis, traités en temps réel et enregistrés. De manière générale, l'identification acoustique effectuée en permanence a été vérifiée par les prélèvements de sédiments; seulement quatre stations ont été mal identifiées, et l'algorithme de reconnaissance a été depuis lors corrigé en conséquence. Les signaux enregistrés ont été rejoués en laboratoire, pour une analyse systématique du taux de réussite du système d'identification. Un taux allant de 70 à $90 \%$ selon les conditions de mesures à étè enregistré sur l'ensemble des échos traités. Les conditions expérimentales les plus pénalisantes correspondent à la présence de bulles sous la coque du navire, soit à l'existence d'un roulis important, qu'il convient soit de compenser à l'aide d'un matériel spécifique, soit plus simplement de filtrer en ne retenant que les signaux réverbérés proches de la verticale.

\section{Conclusion}

La méthơde présentée ici permet d'identifier avec une bonne sûreté les sédiments du plateau continental à l'intérieur d'une classification restreinte; elle est adaptable à $n$ 'importe quel type de sondeur a impulsion courte muni d'un dispositif de numérisation des échos. Elle ne nécessite que la connaissance a priori des caractéristiques du sondeur et du signal utilisés, et $\mathrm{n}^{\prime} \mathrm{a}$ pas besoin de phase d'apprentissage et de calibration. La suite de nos travaux portera sur l'exploitation de cette méthode pour des sondeurs à plusieurs faisceaux, et à l'extension de $1 \mathrm{a}$ méthode à $1^{\prime}$ identification des sédiments des grands fonds.

REFERENCES:

[1] L.Brekhovskikh \& Yu.Lysanov, "Fundamentals of ocean acoustics", Springer Verlag (1982)

[2] A.N.Ivakin \& Yu.P.Lysanov, "Underwater sound scattering by volume inhomogeneities of a bottom medium by a rough surface". Sov. Phys. Acoust. 27.(3) May-June 1981. 

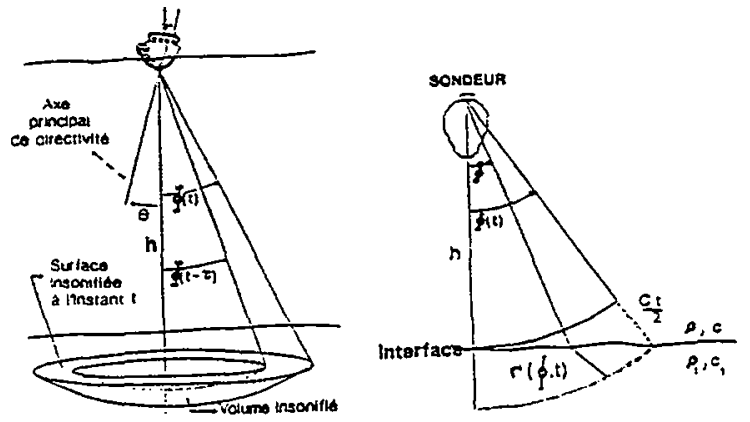

Fig.1. Geometrie de la réverbération d'un signal de sondeur en fonction du temps.

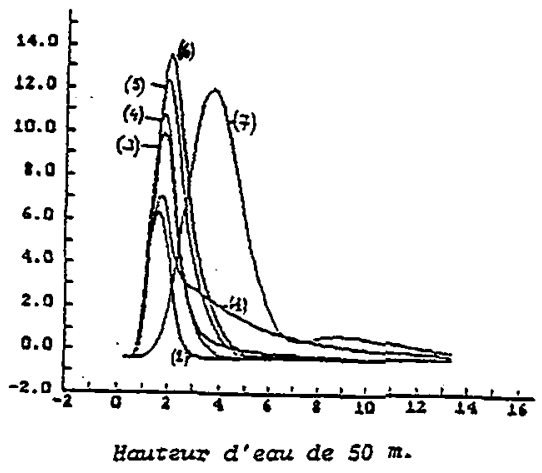

Fig.2. Exemple d'emeloppes temporelles modélisées pour 7 types de fond a $38 \mathrm{kHz}$, impulsion simusoidale de $1 \mathrm{~ms}$, owverture 'a $-3 d B$ de $13^{\circ}$ : L'echantillonnage temporel est de $1,3.10^{-4}$ secondes.

Vase molie (1), Vase (2), Vase-sable (3), Sable très fin (4), Sabie (5), Grovier (6), Roche (7).

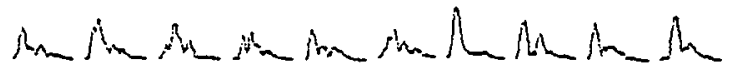

Vase molle. $h=32 \mathrm{~m}, \mathrm{j}=38 \mathrm{kHz}, T=0,5 \mathrm{~ms}$, impulsion sinusożdale.

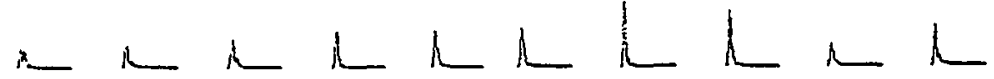

Salie fin $h=52 \mathrm{~m}, f=38 \mathrm{kHz}, T=1,0 \mathrm{~ms}$, impulsion simusoïdale.

L

Gravier. $\quad h=47 \mathrm{~m}, f=38 \mathrm{kHz}, T=1,0 \mathrm{~ms}$, impulsion sinusoiadale.

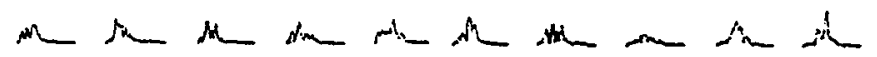

Roche. $\quad h=27 \mathrm{~m}, \rho=38 \mathrm{kH} z, T=0,5 \mathrm{~ms}$, impulsion sinusoïacle.

fig.3 Série d'echos bruts enregistrés sur plusieurs types de fond.

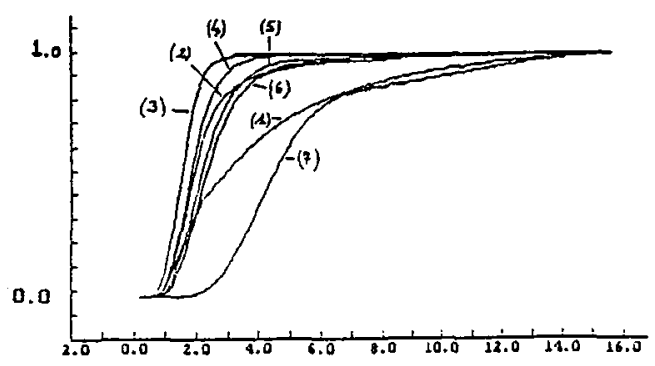

fig.4 Exemple d'enveloppes cumulées normees: $h=50 \mathrm{~m}, T=1,0 \mathrm{~ms}, f=38 \mathrm{kHz}$, impulsion sinusoïdale. 